\title{
Sub-barrier capture with quantum diffusion approach: actinide-based reactions
}

\author{
V.V.Sargsyan ${ }^{1}$, G.G.Adamian ${ }^{1,2}$, N.V.Antonenko ${ }^{1}$, W.Scheid ${ }^{3}$, and H.Q.Zhang ${ }^{4}$ \\ ${ }^{1}$ Joint Institute for Nuclear Research, 141980 Dubna, Russia \\ ${ }^{2}$ Institute of Nuclear Physics, 702132 Tashkent, Uzbekistan \\ ${ }^{3}$ Institut für Theoretische Physik der Justus-Liebig-Universität, D-35392 Giessen, Germany \\ ${ }^{4}$ China Institute of Atomic Energy, Post Office Box 275, Beijing 102413, China
}

(Dated: May 17, 2021)

\begin{abstract}
With the quantum diffusion approach the behavior of capture cross sections and mean-square angular momenta of captured systems are revealed in the reactions with deformed nuclei at subbarrier energies. The calculated results are in a good agreement with existing experimental data. With decreasing bombarding energy under the barrier the external turning point of the nucleusnucleus potential leaves the region of short-range nuclear interaction and action of friction. Because of this change of the regime of interaction, an unexpected enhancement of the capture cross section is expected at bombarding energies far below the Coulomb barrier. This effect is shown its worth in the dependence of mean-square angular momentum of captured system on the bombarding energy. From the comparison of calculated and experimental capture cross sections, the importance of quasifission near the entrance channel is shown for the actinide-based reactions leading to superheavy nuclei.
\end{abstract}

PACS numbers: 25.70.Ji, 24.10.Eq, 03.65.-w

Key words: astrophysical $S$-factor; dissipative dynamics; sub-barrier capture

\section{INTRODUCTION}

The measurement of excitation functions down to the extreme sub-barrier energy region is important for studying the nucleus-nucleus interaction as well as the coupling of relative motion with other degrees of freedom, and very little data exist on the fusion, fission and capture cross sections at extreme sub-barrier energies [1 17]. The experimental data obtained are of interest for solving astrophysical problems related to nuclear synthesis. Indications for an enhancement of the $S$ factor, $S=E_{\text {c.m. }} \sigma \exp (2 \pi \eta)$ [18, 19], where $\eta\left(E_{\text {c.m. }}\right)=$ $Z_{1} Z_{2} e^{2} \sqrt{\mu /\left(2 \hbar^{2} E_{\text {c.m. }}\right)}$ is the Sommerfeld parameter, at energies $E_{\text {c.m. }}$. below the Coulomb barrier have been found in Refs. 7, 10, 13]. However, its origin is still under discussion.

To clarify the behavior of capture cross sections at subbarrier energies, a further development of the theoretical methods is required [20]. The conventional coupledchannel approach with realistic set of parameters is not able to describe the capture cross sections either below or above the Coulomb barrier [13]. The use of a quite shallow nucleus-nucleus potential 21] with an adjusted repulsive core considerably improves the agreement between the calculated and experimental data. Besides the coupling with collective excitations, the dissipation, which is simulated by an imaginary potential in Ref. 21] or by damping in each channel in Ref. 22], seems to be important.

The quantum diffusion approach based on the quantum master-equation for the reduced density matrix has been suggested in Ref. 23]. This model takes into consideration the fluctuation and dissipation effects in collisions of heavy ions which model the coupling with various channels. As demonstrated in Ref. [23], this approach is successful for describing the capture cross sections at energies near and below the Coulomb barrier for interacting spherical nuclei. An unexpected enhancement of the capture cross section at bombarding energies far below the Coulomb barrier has been predicted in 23]. This effect is related to the switching off of the nuclear interaction at the external turning point $r_{e x}$ (Fig. 1). If the colliding nuclei approach the distance $R_{\text {int }}$ between their centers, the nuclear forces start to act in addition to the Coulomb interaction. Thus, at $R<R_{\text {int }}$ the relative motion is coupled strongly with other degrees of freedom. At $R>R_{\text {int }}$ the relative motion is almost independent of the internal degrees of freedom. Depending on whether the value of $r_{e x}$ is larger or smaller than the interaction radius $R_{\text {int }}$, the impact of coupling with other degrees of freedom upon the barrier passage seems to be different.

In the present paper we apply the approach of Ref. 23] to the description of the capture process of deformed nuclei in a wide energy interval including the extreme sub-barrier region. The used formalism is presented in Sect. II. The results of our calculations for the reactions ${ }^{16} \mathrm{O},{ }^{19} \mathrm{~F},{ }^{32} \mathrm{~S},{ }^{48} \mathrm{Ca}+{ }^{232} \mathrm{Th}$, ${ }^{4} \mathrm{He},{ }^{16} \mathrm{O},{ }^{20} \mathrm{Ne},{ }^{30} \mathrm{Si},{ }^{36} \mathrm{~S},{ }^{48} \mathrm{Ca}+{ }^{238} \mathrm{U},{ }^{36} \mathrm{~S},{ }^{48} \mathrm{Ca},{ }^{50} \mathrm{Ti}+{ }^{244} \mathrm{Pu}$, ${ }^{48} \mathrm{Ca}+{ }^{246,248} \mathrm{Cm}$, and ${ }^{36} \mathrm{~S}+{ }^{248} \mathrm{Cm}$ are discussed in Sect. III. The conclusions are given in Sect. IV.

\section{MODEL}

\section{A. The nucleus-nucleus potential}

The potential describing the interaction of two nuclei can be written in the form [24]

$$
\begin{aligned}
V\left(R, Z_{i}, A_{i}, \theta_{i}, J\right) & =V_{C}\left(R, Z_{i}, A_{i}, \theta_{i}, J\right) \\
& +V_{N}\left(R, Z_{i}, A_{i}, \theta_{i}, J\right)+\frac{\hbar^{2} J(J+1)}{2 \mu R^{2}},
\end{aligned}
$$



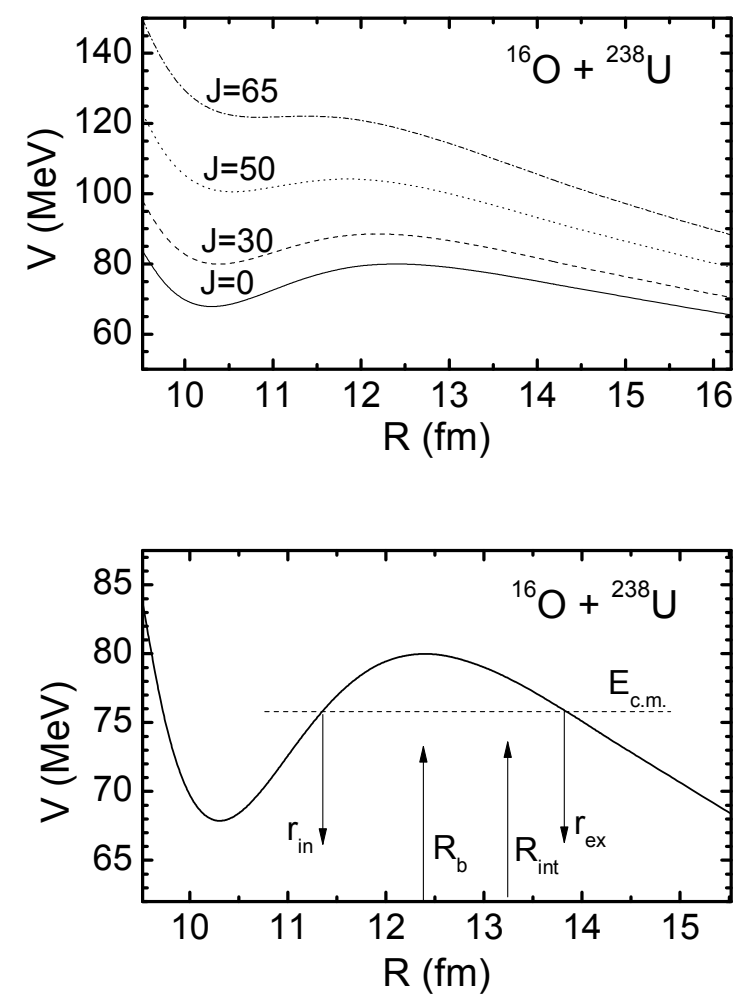

FIG. 1: (Upper part) The nucleus-nucleus potentials calculated at $J=0$ (solid curve), 30 (dashed curve), 50 (dotted curve), and 65 (dash-dotted curve) for the ${ }^{16} \mathrm{O}+{ }^{238} \mathrm{U}$ reaction. The interacting nuclei are assumed to be spherical in the calculation. (Lower part) The position $R_{b}$ of the Coulomb barrier, radius of interaction $R_{\text {int }}$, and external and internal turning points for some values of $E_{\text {c.m. }}$. are indicated at the nucleus-nucleus potential for the same reaction at $J=0$.

where $V_{N}, V_{C}$, and the last summand stand for the nuclear, the Coulomb, and the centrifugal potentials, respectively. The nuclei are proposed to be spherical or deformed. The potential depends on the distance $R$ between the center of mass of two interacting nuclei, mass $A_{i}$ and charge $Z_{i}$ of nuclei $(i=1,2)$, the orientation angles $\theta_{i}$ of the deformed (with the quadrupole deformation parameters $\beta_{i}$ ) nuclei and the angular momentum $J$. The static quadrupole deformation parameters are taken from Ref. 25] for the even-even deformed nuclei. For the nuclear part of the nucleus-nucleus potential, we use the double-folding formalism, in the form

$$
V_{N}=\int \rho_{1}\left(\mathbf{r}_{1}\right) \rho_{2}\left(\mathbf{R}-\mathbf{r}_{2}\right) F\left(\mathbf{r}_{1}-\mathbf{r}_{2}\right) d \mathbf{r}_{1} d \mathbf{r}_{\mathbf{2}}
$$

where $F\left(\mathbf{r}_{\mathbf{1}}-\mathbf{r}_{\mathbf{2}}\right)=C_{0}\left[F_{\text {in }} \frac{\rho_{0}\left(\mathbf{r}_{1}\right)}{\rho_{00}}+F_{\text {ex }}\left(1-\frac{\rho_{0}\left(\mathbf{r}_{1}\right)}{\rho_{00}}\right)\right] \delta\left(\mathbf{r}_{1}-\mathbf{r}_{\mathbf{2}}\right)$ is the density-dependent effective nucleon-nucleon interaction and $\rho_{0}(\mathbf{r})=\rho_{1}(\mathbf{r})+\rho_{2}(\mathbf{R}-\mathbf{r}), F_{\text {in,ex }}=f_{\text {in,ex }}+$ $f_{\text {in,ex }}^{\prime} \frac{\left(N_{1}-Z_{1}\right)\left(N_{2}-Z_{2}\right)}{\left(N_{1}+Z_{1}\right)\left(N_{2}+Z_{2}\right)}$. Here, $\rho_{i}\left(\mathbf{r}_{\mathbf{i}}\right)$ and $N_{i}$ are the nucleon densities and neutron numbers of the light and the heavy nuclei of the dinuclear system, respectively. Our calculations are performed with the following set of parameters: $C_{0}=300$ $\mathrm{MeV} \mathrm{fm}{ }^{3}, f_{\text {in }}=0.09, f_{\text {ex }}=-2.59, f_{\text {in }}^{\prime}=0.42, f_{\text {ex }}^{\prime}=0.54$ and $\rho_{00}=0.17 \mathrm{fm}^{-3}$ [24]. The densities of the nuclei are taken in the two-parameter symmetrized Woods-Saxon form with the nuclear radius parameter $r_{0}=1.15 \mathrm{fm}$ (for the nuclei with $A_{i} \geq 16$ ) and the diffuseness parameter $a$ depending on the charge and mass numbers of the nucleus [24]. We use $a=$ $0.53 \mathrm{fm}$ for the lighter nuclei ${ }^{16} \mathrm{O}$ and ${ }^{19} \mathrm{~F}, a=0.55 \mathrm{fm}$ for the intermediate nuclei $\left({ }^{20} \mathrm{Ne},{ }^{26} \mathrm{Mg},{ }^{30} \mathrm{Si},{ }^{32,34,36} \mathrm{~S},{ }^{40,48} \mathrm{Ca}\right.$, ${ }^{50} \mathrm{Ti}$ ), and $a=0.56 \mathrm{fm}$ for the actinides. For the ${ }^{4} \mathrm{He}$ nucleus $r_{0}=1.02 \mathrm{fm}$ and $a=0.48 \mathrm{fm}$.

The Coulomb interaction of two deformed nuclei has the following form:

$$
\begin{aligned}
& V_{C}\left(R, Z_{i}, A_{i}, \theta_{i}, J\right)=\frac{Z_{1} Z_{2} e^{2}}{R} \\
+ & \left(\frac{9}{20 \pi}\right)^{1 / 2} \frac{Z_{1} Z_{2} e^{2}}{R^{3}} \sum_{i=1,2} R_{i}^{2} \beta_{i}\left[1+\frac{2}{7}\left(\frac{5}{\pi}\right)^{1 / 2} \beta_{i}\right] \\
\times & P_{2}\left(\cos \theta_{i}\right),
\end{aligned}
$$

where $P_{2}\left(\cos \theta_{i}\right)$ is the Legendre polynomial.

In Fig. 1 there is shown the nucleus-nucleus potential $V$ for the ${ }^{16} \mathrm{O}+{ }^{238} \mathrm{U}$ reaction (for simplicity, ${ }^{238} \mathrm{U}$ is assumed to be spherical) which has a pocket. With increasing centrifugal part of the potential the pocket depth becomes smaller, while the position of the pocket minimum moves towards the barrier at the position of the Coulomb barrier $R=R_{b} \approx R_{1}+R_{2}+2$ fm, where $R_{i}=1.15 A_{i}^{1 / 3}$ are the radii of colliding nuclei. This pocket is washed out at large angular momenta $J>65$. Thus, only a limited part of angular momenta contributes to the capture process.

For the reactions ${ }^{36} \mathrm{~S}+{ }^{238} \mathrm{U}$ and ${ }^{16} \mathrm{O}+{ }^{238} \mathrm{U}$ (Fig. 2), the dependence of the potential energy on the orientation of the prolate deformed nucleus ${ }^{238} \mathrm{U}$ is shown. The lowest Coulomb barriers are associated with collisions of the projectile nucleus with the tips of the target nucleus, while the highest barriers correspond to collisions with the sides of the target nucleus. The difference of the Coulomb barriers for the sphere-pole and sphere-side orientations is about $16 \mathrm{MeV}(8 \mathrm{MeV})$ for the ${ }^{36} \mathrm{~S}$ $+{ }^{238} \mathrm{U}\left({ }^{16} \mathrm{O}+{ }^{238} \mathrm{U}\right)$ system.

\section{B. Capture cross section}

The capture cross section is a sum of partial capture cross sections

$$
\begin{aligned}
\sigma_{\text {cap }}\left(E_{\text {c.m. }}\right) & =\sum_{J} \sigma_{\text {cap }}\left(E_{\text {c.m. }}, J\right)= \\
& =\pi \lambda^{2} \sum_{J}(2 J+1) \int_{0}^{\pi / 2} d \theta_{1} \sin \left(\theta_{1}\right) \\
& \times \int_{0}^{\pi / 2} d \theta_{2} \sin \left(\theta_{2}\right) P_{\text {cap }}\left(E_{\text {c.m. }}, J, \theta_{1}, \theta_{2}\right),
\end{aligned}
$$

where $\lambda^{2}=\hbar^{2} /\left(2 \mu E_{\text {c.m. }}\right)$ is the reduced de Broglie wavelength, $\mu=m_{0} A_{1} A_{2} /\left(A_{1}+A_{2}\right)$ is the reduced mass ( $m_{0}$ is the nucleon mass), and the summation is over the possible values of angular momentum $J$ at a given bombarding energy $E_{\text {c.m. }}$. Knowing the potential of the interacting nuclei for each orientation, one can obtain the partial capture probability $P_{\text {cap }}$ which is defined by the passing probability of the potential barrier in the relative distance $R$ coordinate at a given $J$.

The value of $P_{\text {cap }}$ is obtained by integrating the propagator $G$ from the initial state $\left(R_{0}, P_{0}\right)$ at time $t=0$ to the final state 

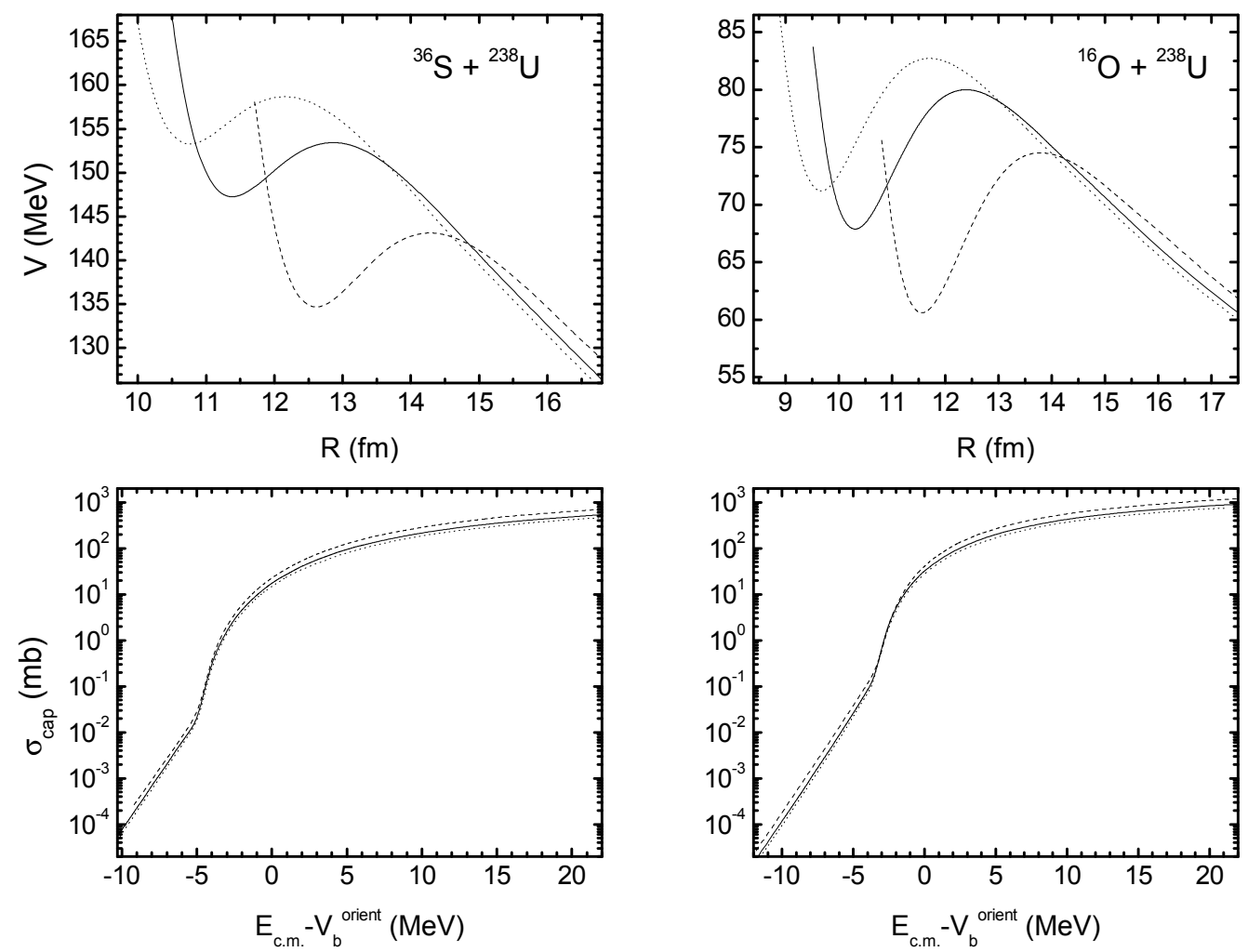

FIG. 2: (Upper part) The nucleus-nucleus potentials calculated at $J=0$ for the reactions ${ }^{36} \mathrm{~S}+{ }^{238} \mathrm{U}$ and ${ }^{16} \mathrm{O}+{ }^{238} \mathrm{U} \cdot(\mathrm{Lower}$ part) The dependence of the capture cross section for nuclei colliding with a fixed orientation on $E_{\mathrm{c} . \mathrm{m} .}-V_{b}^{\text {orient }}$ where $V_{b}^{\text {orient }}$ is the height of the Coulomb barrier for certain orientations. The results of calculations for the sphere-sphere (the interacting nuclei are spherical), sphere-pole and sphere-side configurations are shown by solid, dashed and dotted lines, respectively. The static quadrupole deformation parameters are: $\beta_{2}\left({ }^{238} \mathrm{U}\right)=0.286$ and $\beta_{1}\left({ }^{16} \mathrm{O}\right)=\beta_{1}\left({ }^{36} \mathrm{~S}\right)=0$.

$(R, P)$ at time $t(P$ is a momentum):

$$
\begin{aligned}
P_{\text {cap }} & =\lim _{t \rightarrow \infty} \int_{-\infty}^{r_{\text {in }}} d R \int_{-\infty}^{\infty} d P G\left(R, P, t \mid R_{0}, P_{0}, 0\right) \\
& =\lim _{t \rightarrow \infty} \frac{1}{2} \operatorname{erfc}\left[\frac{-r_{\text {in }}+\overline{R(t)}}{\sqrt{\Sigma_{R R}(t)}}\right] .
\end{aligned}
$$

The second line in (5) is obtained by using the propagator $G=\pi^{-1}\left|\operatorname{det} \boldsymbol{\Sigma}^{-1}\right|^{1 / 2} \exp \left(-\mathbf{q}^{T} \boldsymbol{\Sigma}^{-1}\right)\left(\mathbf{q}^{T}=\left[q_{R}, q_{P}\right]\right.$,

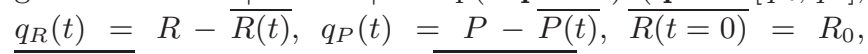
$\overline{P(t=0)}=P_{0}, \quad \Sigma_{k k^{\prime}}(t)=2 \overline{q_{k}(t) q_{k^{\prime}}(t)}, \Sigma_{k k^{\prime}}(t=0)=0$, $\left.k, k^{\prime}=R, P\right)$ calculated in Ref. [26] for an inverted oscillator which approximates the nucleus-nucleus potential $V$ in the variable $R$. The frequency $\omega$ of this oscillator with an internal turning point $r_{\text {in }}$ is defined from the condition of equality of the classical actions of approximated and realistic potential barriers of the same hight at given $J$. It should be noted that the passage through the Coulomb barrier approximated by a parabola has been previously studied in Refs. 27 31]. This approximation is well justified for the reactions and energy range, which are here considered. Finally, one can find the expression for the capture probability:

$$
P_{\text {cap }}=\frac{1}{2} \operatorname{erfc}\left[\left(\frac{\pi s_{1}\left(\gamma-s_{1}\right)}{2 \mu\left(\omega_{0}^{2}-s_{1}^{2}\right)}\right)^{1 / 2} \frac{\mu \omega_{0}^{2} R_{0} / s_{1}+P_{0}}{\left[\gamma \ln \left(\gamma / s_{1}\right)\right]^{1 / 2}}\right],
$$

where $\gamma$ is the internal-excitation width, $\omega_{0}^{2}=\omega^{2}\{1-$ $\left.\hbar \tilde{\lambda} \gamma /\left[\mu\left(s_{1}+\gamma\right)\left(s_{2}+\gamma\right)\right]\right\}$ is the renormalized frequency in the Markovian limit, the value of $\tilde{\lambda}$ is related to the strength of linear coupling in coordinates between collective and internal subsystems. The $s_{i}$ are the real roots $\left(s_{1} \geq 0>s_{2} \geq s_{3}\right)$ of the following equation:

$$
(s+\gamma)\left(s^{2}-\omega_{0}^{2}\right)+\hbar \tilde{\lambda} \gamma s / \mu=0
$$

The details of the used formalism are presented in [23]. We have to mention that most of the quantum-mechanical, dissipative effects and non-Markovian effects accompanying the passage through the potential barrier are taken into consideration in our formalism [23, 31]. For example, the nonMarkovian effects appear in the calculations through the internal-excitation width $\gamma$.

As shown in 23], the nuclear forces start to play a role at $R_{\text {int }}=R_{b}+1.1 \mathrm{fm}$ where the nucleon density of colliding 


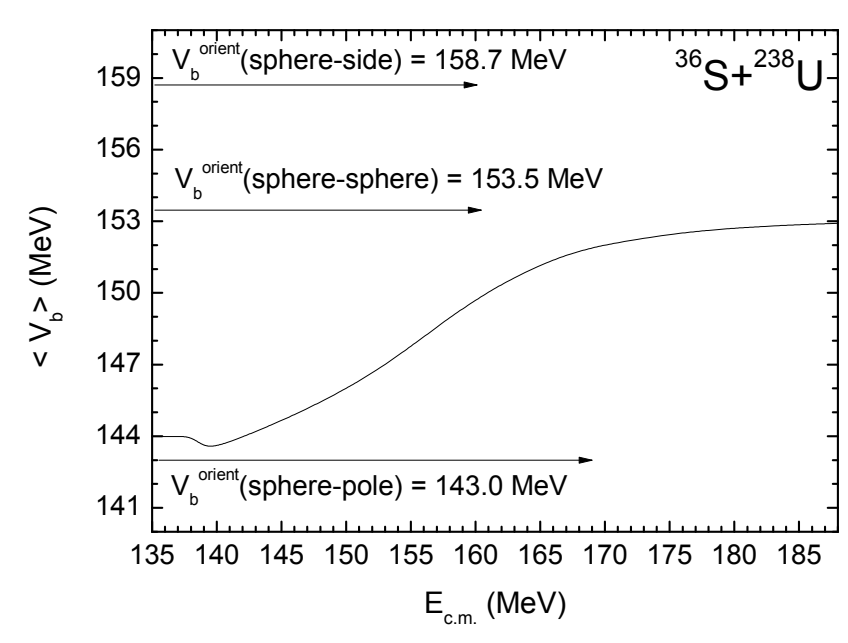

FIG. 3: The calculated value $\left\langle V_{b}\right\rangle$ averaged over the orientations of the heavy deformed nucleus versus $E_{\text {c.m. }}$. for the ${ }^{36} \mathrm{~S}+{ }^{238} \mathrm{U}$ reaction. The values of barriers $V_{b}^{\text {orient }}$ (spherepole) for the sphere-pole configuration, $V_{b}=V_{b}$ (spheresphere $)=V_{b}^{\text {orient }}$ (sphere-sphere) for the sphere-sphere configuration and $V_{b}^{\text {orient }}$ (sphere-side) for the sphere-side configuration are indicated by arrows. The static quadrupole deformation parameters are: $\beta_{2}\left({ }^{238} \mathrm{U}\right)=0.286$ and $\beta_{1}\left({ }^{36} \mathrm{~S}\right)=0$.

nuclei approximately reaches $10 \%$ of the saturation density. If the value of $r_{\mathrm{ex}}$ corresponding to the external turning point is larger than the interaction radius $R_{\text {int }}$, we take $R_{0}=r_{\mathrm{ex}}$ and $P_{0}=0$ in Eq. (6). For $r_{\mathrm{ex}}<R_{\text {int }}$, it is naturally to start our treatment with $R_{0}=R_{\text {int }}$ and $P_{0}$ defined by the kinetic energy at $R=R_{0}$. In this case the friction hinders the classical motion to proceed towards smaller values of $R$. If $P_{0}=0$ at $R_{0}>R_{\text {int }}$, the friction almost does not play a role in the transition through the barrier. Thus, two regimes of interaction at sub-barrier energies differ by the action of the nuclear forces and the role of friction at $R=r_{\mathrm{ex}}$.

\section{CALCULATED RESULTS}

Besides the parameters related to the nucleus-nucleus potential, two parameters $\hbar \gamma=32 \mathrm{MeV}$ and the friction coefficient $\hbar \lambda=-\hbar\left(s_{1}+s_{2}\right)=2 \mathrm{MeV}$ are used for calculating the capture probability in reactions with deformed actinides. The value of $\tilde{\lambda}$ is set to obtain this value of $\hbar \lambda$. The most realistic friction coefficients in the range of $\hbar \lambda \approx 1-2 \mathrm{MeV}$ are suggested from the study of deep inelastic and fusion reactions [32]. These values are close to those calculated within the mean field approach [33]. All calculated results presented are obtained with the same set of parameters and are rather insensitive to a reasonable variation of them [23, 28, 31].
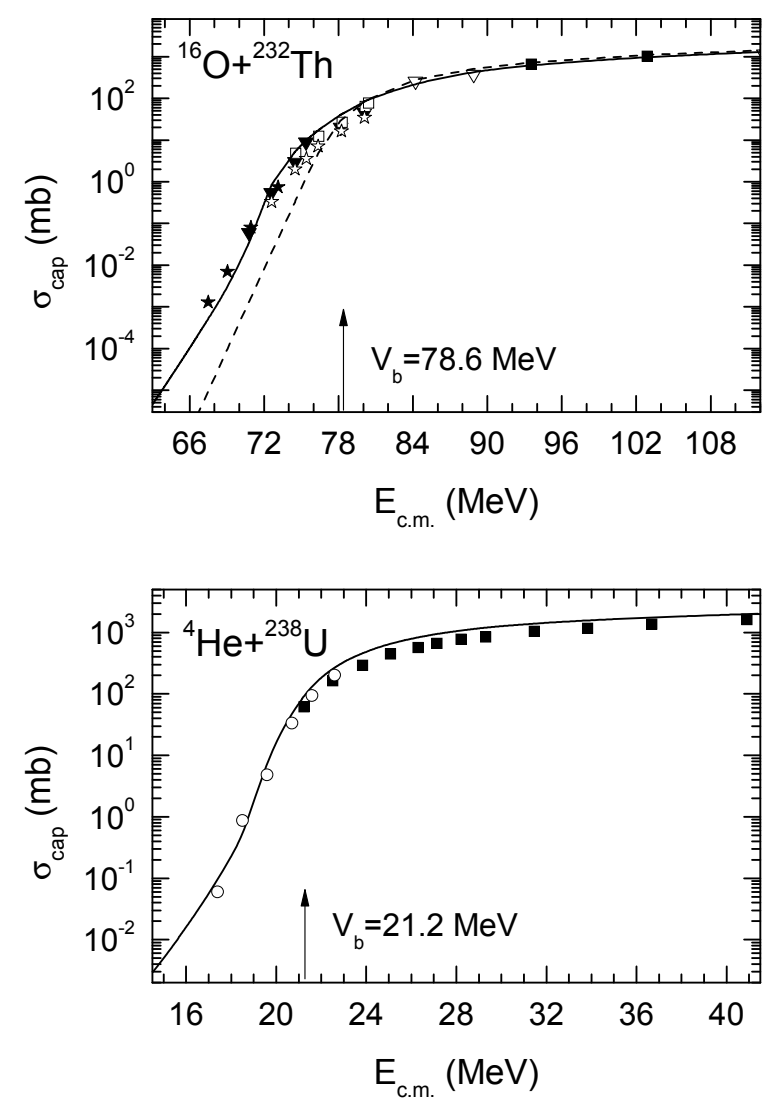

FIG. 4: The calculated capture cross section (solid lines) versus $E_{\text {c.m. }}$. for the reactions ${ }^{16} \mathrm{O}+{ }^{232} \mathrm{Th}$ and ${ }^{4} \mathrm{He}+{ }^{238} \mathrm{U}$ are compared with the available experimental data. The experimental data in the upper part are taken from Refs. 37] (open triangles), 1] (closed triangles), 38] (open squares), 39] (closed squares), [4] (open stars) and [5] (closed stars). The fission cross sections from Refs. [6] and [36] are shown in the lower part by open circles and solid squares, respectively. The value of the Coulomb barrier $V_{b}$ for the spherical nuclei is indicated by arrow. The dashed curve represents the calculation by the Wong's formula (8). The static quadrupole deformation parameters are: $\beta_{2}\left({ }^{238} \mathrm{U}\right)=0.286, \beta_{2}\left({ }^{232} \mathrm{Th}\right)=0.261$ and $\beta_{1}\left({ }^{16} \mathrm{O}\right)=\beta_{1}\left({ }^{4} \mathrm{He}\right)=0$.

\section{A. Effect of orientation}

The influence of orientation of the deformed heavy nucleus on the capture process in the reactions ${ }^{36} \mathrm{~S}+{ }^{238} \mathrm{U}$ and ${ }^{16} \mathrm{O}$ $+{ }^{238} \mathrm{U}$ is studied in Fig. 2. We demonstrate that the capture cross section $\sigma_{c a p}$ at fixed orientation as a function of $E_{\text {c.m. }}-V_{b}^{\text {orient }}$, where $V_{b}^{\text {orient }}$ is the Coulomb barrier for this orientation, is almost independent of the orientation angle $\theta_{2}$.

In Fig. 3 the value of the Coulomb barrier

$$
\begin{aligned}
<V_{b}> & =\frac{\pi \lambda^{2}}{\sigma_{\text {cap }}\left(E_{\text {c.m. }}\right)} \sum_{J}(2 J+1) \int_{0}^{\pi / 2} d \theta_{2} \sin \left(\theta_{2}\right) \\
& \times P_{\text {cap }}\left(E_{\text {c.m. }}, J, \theta_{1}, \theta_{2}\right) V\left(R_{b}, Z_{i}, A_{i}, \theta_{i}, J\right)
\end{aligned}
$$



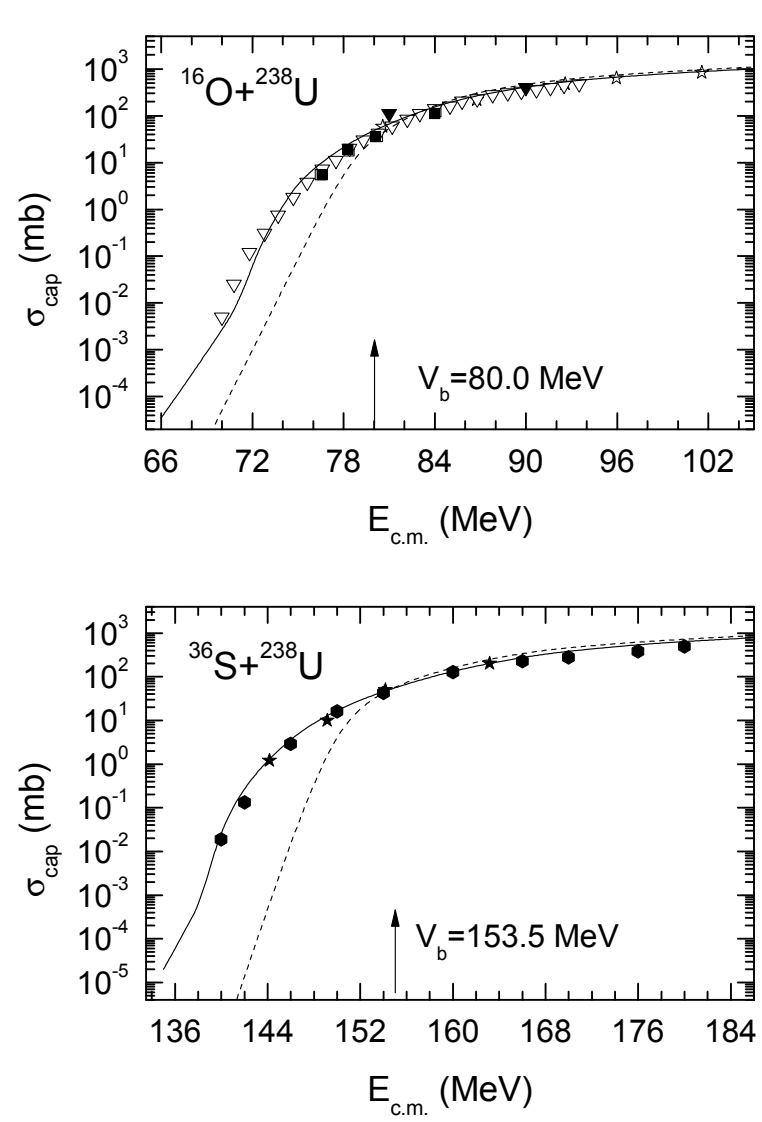

FIG. 5: The same as in Fig. 4, but for the reactions ${ }^{16} \mathrm{O}$ $+{ }^{238} \mathrm{U}$ and ${ }^{36} \mathrm{~S}+{ }^{238} \mathrm{U}$. The experimental cross sections are taken from Refs. 9] (open triangles), 35] (closed triangles), [4] (open squares), 2] (closed squares), [36] (open stars), 16] (closed stars), and [14] (rhombuses). The dashed curve represents the calculation by the Wong's formula (8). The static quadrupole deformation parameters are: $\beta_{2}\left({ }^{238} \mathrm{U}\right)=0.286$ and $\beta_{1}\left({ }^{16} \mathrm{O}\right)=\beta_{1}\left({ }^{36} \mathrm{~S}\right)=0$.

averaged over all possible orientations of the heavy nucleus versus $E_{\text {c.m. }}$. is shown for the ${ }^{36} \mathrm{~S}+{ }^{238} \mathrm{U}$ reaction. With increasing (decreasing) $E_{\text {c.m. }}$. the value of $\left\langle V_{b}\right\rangle$ approaches the value of the Coulomb barrier for the sphere-sphere configuration (for the sphere-pole configuration). The influence of deformation on the capture cross section is very weak already at bombarding energies about $15 \mathrm{MeV}$ above the Coulomb barrier corresponding to spherical nuclei.

\section{B. Comparison with experimental data and predictions}

In Figs. 4-6 the calculated capture cross sections for the reactions ${ }^{16} \mathrm{O},{ }^{19} \mathrm{~F},{ }^{32} \mathrm{~S}+{ }^{232} \mathrm{Th}$ and ${ }^{4} \mathrm{He},{ }^{16} \mathrm{O},{ }^{30} \mathrm{Si},{ }^{32,36} \mathrm{~S}+{ }^{238} \mathrm{U}$ are in a rather good agreement with the available experimental data [1, 2, 4 6, 9, 11, 14 16, 35 40]. Because of the uncertainties in the definition of the deformation of the light nucleus and in the experimental data [11, 40] in Fig. 6, we show the calculated results for the ${ }^{30} \mathrm{Si}+{ }^{238} \mathrm{U}$ reaction with
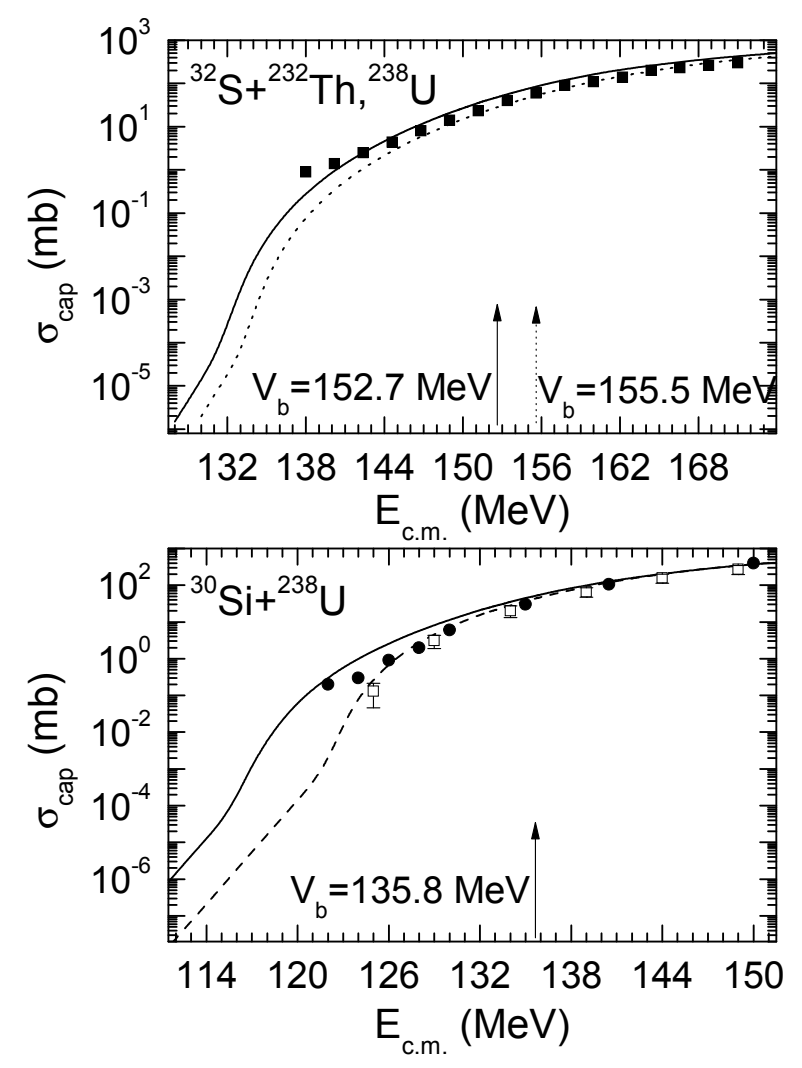

FIG. 6: The same as in Fig. 4, but for the reactions ${ }^{32} \mathrm{~S}$ $+{ }^{232} \mathrm{Th}$ (solid line), ${ }^{32} \mathrm{~S}+{ }^{238} \mathrm{U}$ (dotted line) and ${ }^{30} \mathrm{Si}+$ ${ }^{238} \mathrm{U}$. The experimental data are taken from Refs. 15$]\left({ }^{32} \mathrm{~S}\right.$ $+{ }^{232} \mathrm{Th}$, solid squares), [11] (solid circles) and 40] (open squares). The static quadrupole deformation parameters are: $\beta_{2}\left({ }^{238} \mathrm{U}\right)=0.286, \beta_{2}\left({ }^{232} \mathrm{Th}\right)=0.261, \beta_{1}\left({ }^{32} \mathrm{~S}\right)=0.312$ and $\beta_{1}\left({ }^{30} \mathrm{Si}\right)=0.315$. For the ${ }^{30} \mathrm{Si}+{ }^{238} \mathrm{U}$ reaction, the results of calculations with $\beta_{1}\left({ }^{30} \mathrm{Si}\right)=0$ (the predictions of the meanfield and macroscopic-microscopic models) are presented by dashed line in the lower part of the figure.

$\beta_{1}\left({ }^{30} \mathrm{Si}\right)$ from Ref. [25] as well as with $\beta_{1}\left({ }^{30} \mathrm{Si}\right)=0$ (lower part of Fig. 6). Note that $\beta_{1}\left({ }^{30} \mathrm{Si}\right)=0$ for the ground state were predicted within the mean-field and macroscopic-microscopic models.

In Fig. 7 (upper part) we are not able to describe well the data of Ref. [5] for the ${ }^{19} \mathrm{~F}+{ }^{232} \mathrm{Th}$ reaction at $E_{\text {c.m. }}<$ $74 \mathrm{MeV}$, even by varying the static quadrupole deformation parameters $\beta_{1}$ of ${ }^{19} \mathrm{~F}$. However, the deviations of the solid curve in the upper part of Fig. 7 from the experimental data are within the uncertainty of these data. Note that the value of $\beta_{1}$ mainly influences the slope of curve at $E_{\text {c.m. }}<V_{b}$ and one can extract the ground state deformation of nucleus from the experimental capture cross section data. For the ${ }^{20} \mathrm{Ne}+$ ${ }^{238} \mathrm{U}$ reaction, the calculated capture cross sections in Fig. 7 are consistent with the experimental data [36] if the latter ones are shifted by $2 \mathrm{MeV}$ to lower energies. For the ${ }^{20} \mathrm{Ne}$ nucleus, the experimental quadrupole deformation parameter $\beta_{1}=0.727$ related in Ref. [25] to the first $2^{+}$state seems to 

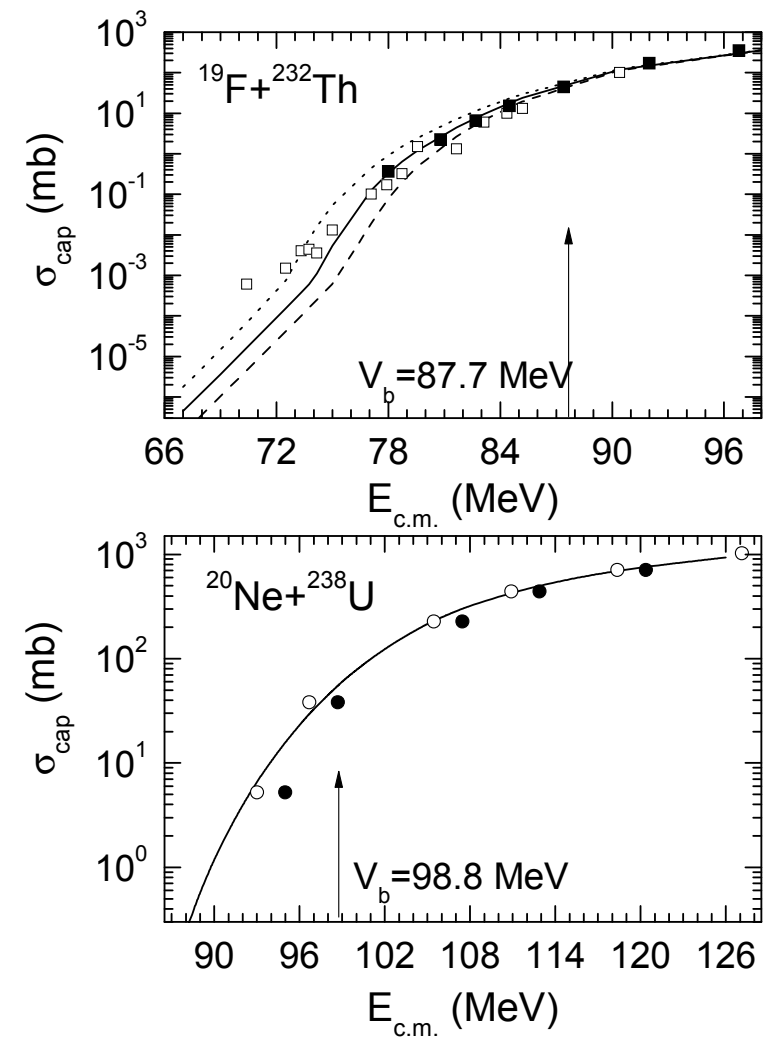

FIG. 7: The same as in Fig. 4, but for the reactions ${ }^{19} \mathrm{~F}+$ ${ }^{232} \mathrm{Th}$ and ${ }^{20} \mathrm{Ne}+{ }^{238} \mathrm{U}$. The experimental data are taken from Refs. 5] (open squares), 44] (closed squares), and [36] (closed circles). The open circles in the lower part are the experimental data from Ref. [36] shifted by $2 \mathrm{MeV}$ to the left. The results of calculations with the static quadrupole deformation parameters of ${ }^{19} \mathrm{~F} \beta_{1}\left({ }^{19} \mathrm{~F}\right)=0.275$ (as in Ref. [41]), 0.41, and 0.55 are shown by the dashed, solid, and dotted lines, respectively. The other static quadrupole deformation parameters are: $\beta_{2}\left({ }^{238} \mathrm{U}\right)=0.286, \beta_{2}\left({ }^{232} \mathrm{Th}\right)=0.261$ and $\beta_{1}\left({ }^{20} \mathrm{Ne}\right)=0.335$.

be unrealistically large and we take $\beta_{1}=0.335$ as predicted in Ref. 41]. The capture cross sections for the reactions ${ }^{32} \mathrm{~S}$ $+{ }^{238} \mathrm{U}$ and ${ }^{36} \mathrm{~S}+{ }^{244} \mathrm{Pu},{ }^{248} \mathrm{Cm}$ are shown in Figs. 6 and 8 , respectively.

One can see in Figs. 4-8 that there is a sharp fall-off of the cross sections just under the Coulomb barrier corresponding to undeformed nuclei. With decreasing $E_{\text {c.m. }}$. up to about 8-10 MeV (when the projectile is spherical) and $15-20 \mathrm{MeV}$ (when both projectile and target are deformed nuclei) below the Coulomb barrier the regime of interaction is changed because at the external turning point the colliding nuclei do not reach the region of nuclear interaction where the friction plays a role. As result, at smaller $E_{\text {c.m. }}$. the cross sections fall with a smaller rate. With larger values of $R_{\text {int }}$ the change of fall rate occurs at smaller $E_{\text {c.m. }}$. However, the uncertainty in the definition of $R_{\text {int }}$ is rather small. Therefore, an effect of the change of fall rate of sub-barrier capture cross section should be in the data if we assume that the friction starts
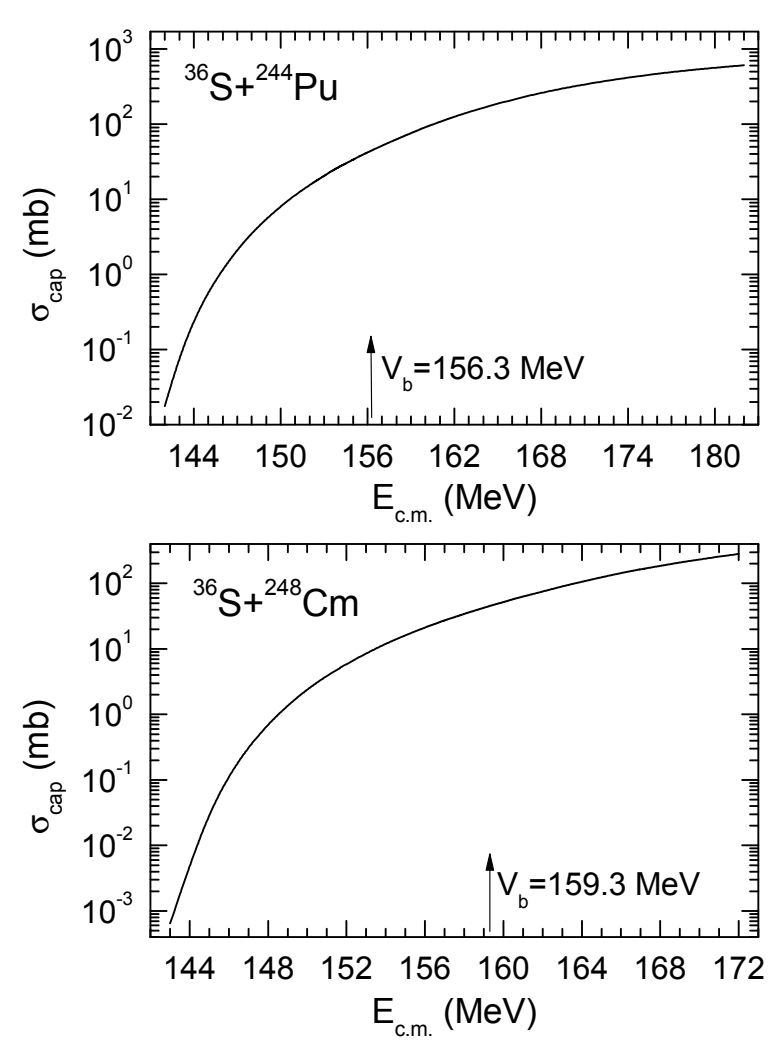

FIG. 8: The predicted capture cross sections for the reactions ${ }^{36} \mathrm{~S}+{ }^{244} \mathrm{Pu},{ }^{248} \mathrm{Cm}$. The static quadrupole deformation parameters are: $\beta_{2}\left({ }^{244} \mathrm{Pu}\right)=0.293, \beta_{2}\left({ }^{248} \mathrm{Cm}\right)=0.297$ and $\beta_{1}\left({ }^{36} \mathrm{~S}\right)=0$.

to act only when the colliding nuclei approach the barrier. Note that at energies of $10-20 \mathrm{MeV}$ below the barrier the experimental data have still large uncertainties to make a firm experimental conclusion about this effect. The effect seems to be more pronounced in collisions of spherical nuclei, where the regime of interaction is changed at $E_{\text {c.m. }}$. up to about 3-5 $\mathrm{MeV}$ below the Coulomb barrier [23]. The collisions of deformed nuclei occur at various mutual orientations affecting the value of $R_{\text {int }}$.

The well-known Wong formula for the capture cross section is

$$
\begin{aligned}
\sigma\left(E_{\text {c.m. }}\right) & =\frac{R_{b}^{2} \hbar \omega}{2 E_{\text {c.m. }}} \int_{0}^{\pi / 2} d \theta_{1} \sin \theta_{1} \int_{0}^{\pi / 2} d \theta_{2} \sin \theta_{2} \\
& \times \ln \left(1+\exp \left[2 \pi\left(E_{\text {c.m. }}-E_{b}\left(\theta_{1}, \theta_{2}\right)\right) / \hbar \omega\right]\right)
\end{aligned}
$$

where $E_{b}\left(\theta_{1}, \theta_{2}\right)$ is value of the Coulomb barrier which depends on the orientations of the deformed nuclei [44]. As seen from Figs. 4 and 5 (dashed lines) the Wong formula (8) does not reproduce the capture cross section at $E_{\text {c.m. }}<V_{b}$ even taking into consideration the static quadrupole deformation of target-nucleus. 

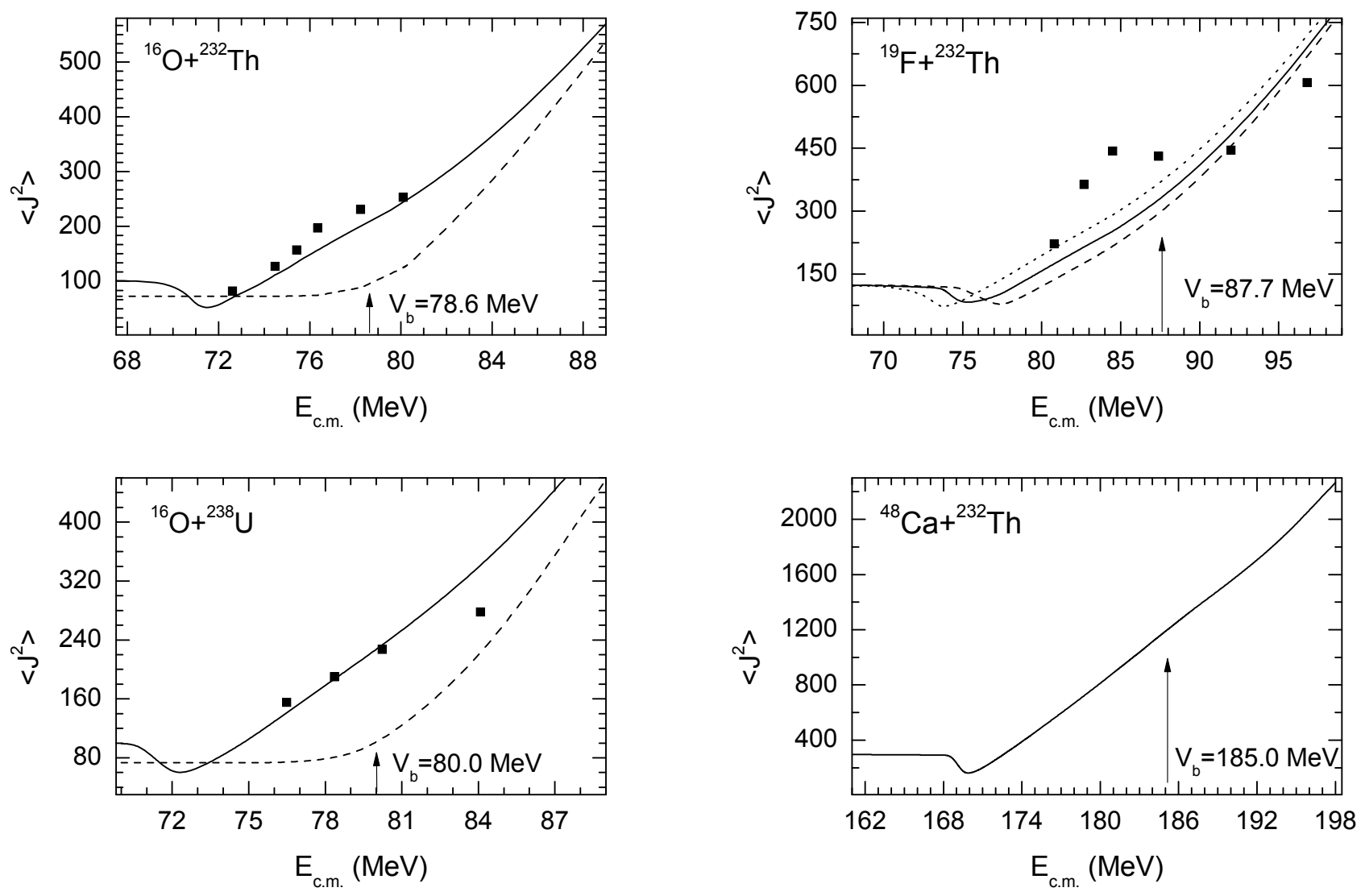

FIG. 9: The calculated mean-square angular momenta versus $E_{\text {c.m. }}$. for the reactions ${ }^{16} \mathrm{O}+{ }^{232} \mathrm{Th},{ }^{238} \mathrm{U}$ are compared with experimental data [4]. The dashed curve represents the calculation by the Eq. (10). The static quadrupole deformation parameters are: $\beta_{2}\left({ }^{238} \mathrm{U}\right)=0.286, \beta_{2}\left({ }^{232} \mathrm{Th}\right)=0.261$ and $\beta_{1}\left({ }^{16} \mathrm{O}\right)=0$. The values of the Coulomb barriers $V_{b}$ corresponding to spherical interacting nuclei are indicated by arrows.

The calculated mean-square angular momenta

$$
\begin{aligned}
\left\langle J^{2}\right\rangle & =\frac{\pi \lambda^{2} \sum_{J} J(J+1)(2 J+1)}{\sigma_{\text {cap }}\left(E_{\text {c.m. }}\right)} \\
& \times \int_{0}^{\pi / 2} d \theta_{1} \sin \left(\theta_{1}\right) \int_{0}^{\pi / 2} d \theta_{2} \sin \left(\theta_{2}\right) P_{\text {cap }}\left(E_{\text {c.m. }}, J, \theta_{1}, \theta_{2}\right)
\end{aligned}
$$

of captured systems versus $E_{\text {c.m. }}$ are presented in Figs. 9-10 for the reactions mentioned above. At energies below the barrier the value of $\left\langle J^{2}\right\rangle$ has a minimum. This minimum depends on the deformations of nuclei and on the factor $Z_{1} \times Z_{2}$. For the reactions ${ }^{16} \mathrm{O}+{ }^{232} \mathrm{Th},{ }^{16} \mathrm{O}+{ }^{238} \mathrm{U},{ }^{19} \mathrm{~F}+{ }^{232} \mathrm{Th}$ and ${ }^{48} \mathrm{Ca}$ $+{ }^{232} \mathrm{Th}$, these minima are about $7,8,12$ and $15 \mathrm{MeV}$ below the corresponding Coulomb barriers, respectively. The experimental data [42] indicate the presence of the minimum as well. On the left-hand side of this minimum the dependence of $\left\langle J^{2}\right\rangle$ on $E_{\text {c.m. }}$. is rather weak. A similar weak dependence

FIG. 10: The same as in Fig. 9, but for the indicated reactions ${ }^{19} \mathrm{~F},{ }^{48} \mathrm{Ca}+{ }^{232} \mathrm{Th}$. The experimental data are taken from Ref. [4]. The results of calculations with quadrupole deformation parameters $\beta_{1}\left({ }^{19} \mathrm{~F}\right)=0.275,0.41$ and 0.55 are shown by the dashed, solid, and dotted lines, respectively. The other static quadrupole deformation parameters are: $\beta_{2}\left({ }^{232} \mathrm{Th}\right)=0.261$ and $\beta_{1}\left({ }^{48} \mathrm{Ca}\right)=0$.

has been found in Refs. [43] in the extreme sub-barrier region. Note that the found behavior of $\left\langle J^{2}\right\rangle$, which is related to the change of the regime of interaction between the colliding nuclei, would affect the angular anisotropy of the products of fission-like fragments following capture. Indeed, the values of $\left\langle J^{2}\right\rangle$ are extracted from data on angular distribution of fission-like fragments [17].

In the Wong model [44] the value of the mean-square angular momentum is determined as

$$
\begin{aligned}
\left\langle J^{2}\right\rangle & =\frac{\mu R_{b}^{2} \hbar \omega}{\pi \hbar^{2}} \int_{0}^{\pi / 2} d \theta_{1} \sin \theta_{1} \int_{0}^{\pi / 2} d \theta_{2} \sin \theta_{2} \\
& \times \frac{-L i_{2}\left(-\exp \left[2 \pi\left(E_{\text {c.m. }}-E_{b}\left(\theta_{1}, \theta_{2}\right)\right) / \hbar \omega\right]\right)}{\ln \left(1+\exp \left[2 \pi\left(E_{\text {c.m. }}-E_{b}\left(\theta_{1}, \theta_{2}\right)\right) / \hbar \omega\right]\right)}
\end{aligned}
$$

Here, the $L i_{2}(z)$ is the polylogarithm function. At $\left.\exp \left[2 \pi\left(E_{\text {c.m. }}-E_{b}\right) / \hbar \omega\right]\right) \ll 1$ (much below the Coulomb barrier $), \frac{-L i_{2}\left(-\exp \left[2 \pi\left(E_{\text {c.m. }}-E_{b}\right) / \hbar \omega\right]\right)}{\ln \left(1+\exp \left[2 \pi\left(E_{\text {c.m. }}-E_{b}\right) / \hbar \omega\right]\right)} \approx 1$ and one can obtain the 

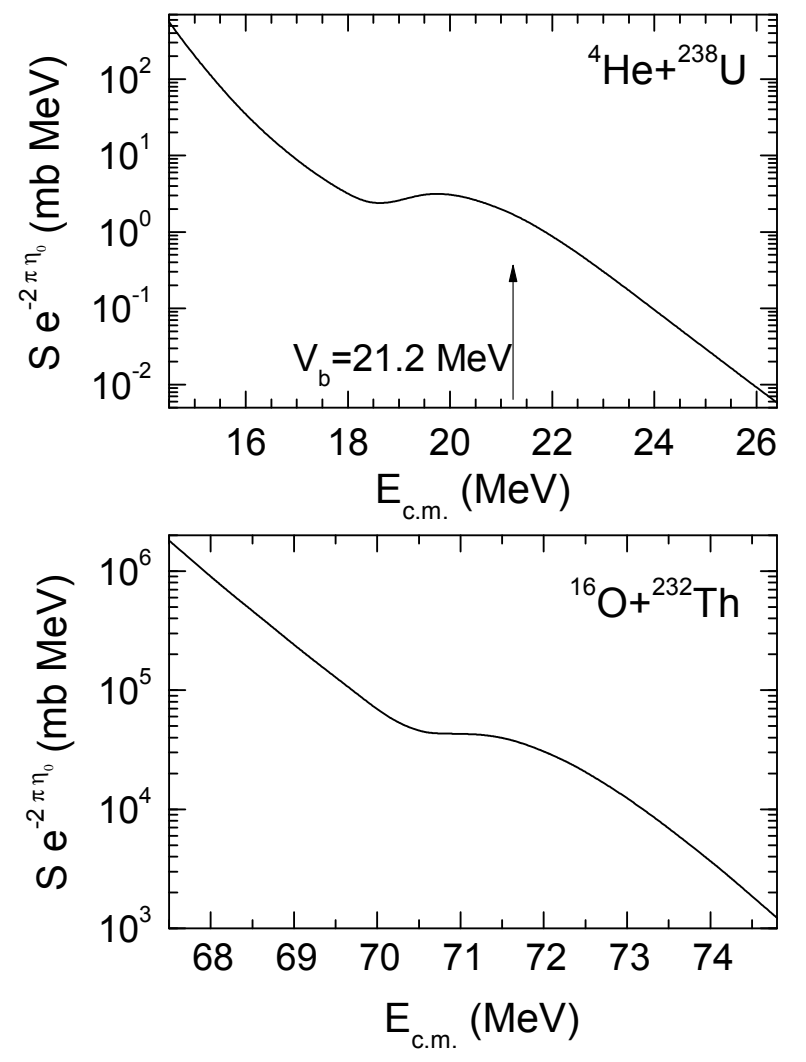

FIG. 11: The calculated values of the astrophysical $S$ factor with $\eta_{0}=\eta\left(E_{\text {c.m. }}=V_{b}\right)$ for the indicated reactions ${ }^{16} \mathrm{O}+{ }^{232} \mathrm{Th}$ and ${ }^{4} \mathrm{He}+{ }^{238} \mathrm{U}$. The values of the Coulomb barriers $V_{b}$ corresponding to the spherical nuclei are 78.6 and 21.2 $\mathrm{MeV}$.

saturation value of the mean-square angular momentum [20]:

$$
\left\langle J^{2}\right\rangle=\frac{\mu R_{b}^{2} \hbar \omega}{\pi \hbar^{2}}
$$

The agreement between $\left\langle J^{2}\right\rangle$ calculated with Eq. (10) and experimental $\left\langle J^{2}\right\rangle$ is not good. At energies below the barrier $\left\langle J^{2}\right\rangle$ has no a minimum (see Fig. 9). However, for the considered reactions the saturation values of $\left\langle J^{2}\right\rangle$ are close to those obtained with our formalism.

\section{Astrophysical factor, L-factor and barrier distribution}

In Figs. 11 and 12 the calculated astrophysical $S$-factors versus $E_{\text {c.m. }}$ are shown for the reactions ${ }^{4} \mathrm{He},{ }^{16} \mathrm{O}+{ }^{238} \mathrm{U}$ and ${ }^{16} \mathrm{O}+{ }^{232} \mathrm{Th}$. The $S$-factor has a maximum for which there are experimental indications in Refs. [7, 10, 21]. After this maximum $S$-factor slightly decreases with decreasing $E_{\text {c.m. }}$. and then starts to increase. This effect seems to be more

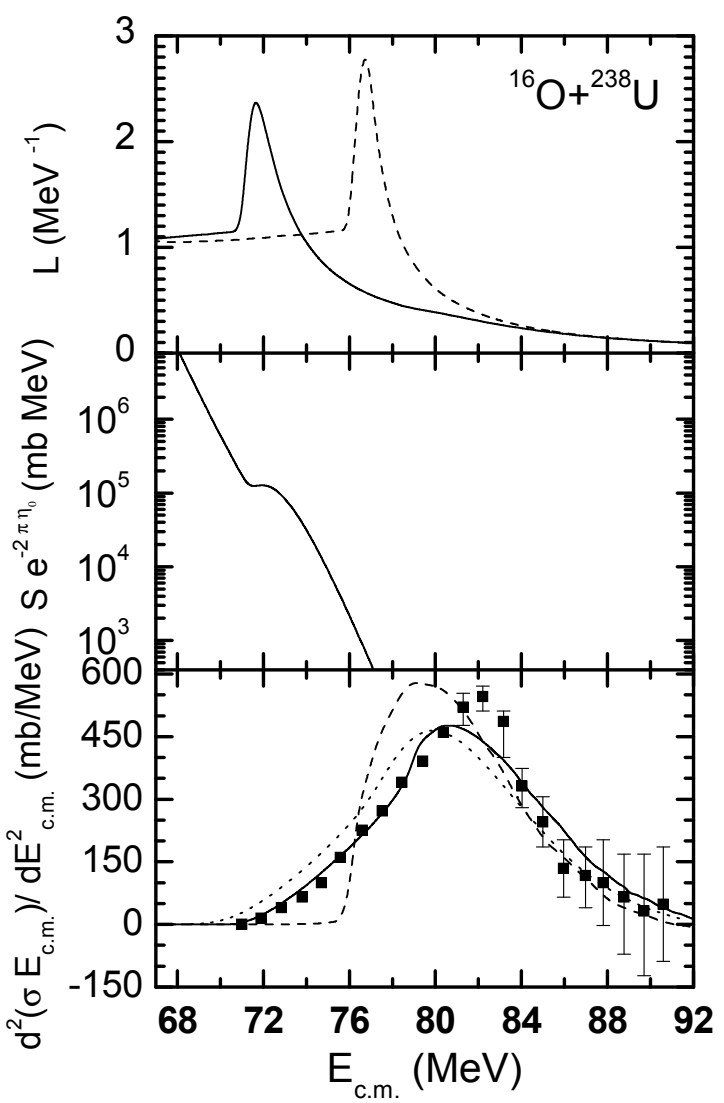

FIG. 12: The calculated values of the astrophysical $S$-factor with $\eta_{0}=\eta\left(E_{\text {c.m. }}=V_{b}\right)$ (middle part), the logarithmic derivative $L$ (upper part) and the fusion barrier distribution $d^{2}\left(E_{\text {c.m. }} \sigma_{\text {cap }}\right) / d E_{\text {c.m. }}^{2}$. (lower part) for the ${ }^{16} \mathrm{O}+{ }^{238} \mathrm{U}$ reaction. The value of $L$ calculated with the assumption of $\beta_{1}\left({ }^{16} \mathrm{O}\right)=\beta_{2}\left({ }^{238} \mathrm{U}\right)=0$ is shown by a dashed line. The solid and dotted lines show the values of $d^{2}\left(E_{\text {c.m. }} \sigma_{\text {cap }}\right) / d E_{\text {c.m. }}^{2}$ calculated with the increments 0.2 and $1.2 \mathrm{MeV}$, respectively. The closed squares are the experimental data of Ref. [50]. The value of the Coulomb barrier $V_{b}$ corresponding to the spherical nuclei is $80 \mathrm{MeV}$.

pronounced in collisions of spherical nuclei [23]. The same behavior has been revealed in Refs. 34] by extracting the $S$-factor from the experimental data.

In Fig. 12, the so-called logarithmic derivative, $L\left(E_{\text {c.m. }}\right)=d\left(\ln \left(E_{\text {c.m. }} \sigma_{\text {cap }}\right)\right) / d E_{\text {c.m. }}$, and the barrier distribution $d^{2}\left(E_{\text {c.m. }} \sigma_{\text {cap }}\right) / d E_{\text {c.m. }}^{2}$. are presented for the ${ }^{16} \mathrm{O}+{ }^{238} \mathrm{U}$ reaction. The logarithmic derivative strongly increases below the barrier and then has a maximum at $E_{\text {c.m. }} \approx V_{b}^{\text {orient }}$ (sphere-pole)-3 MeV (at $E_{\text {c.m. }} \approx V_{b}-3$ $\mathrm{MeV}$ for the case of spherical nuclei). The maximum of $L$ corresponds to the minimum of the $S$-factor.

The barrier distributions calculated with an energy increment $0.2 \mathrm{MeV}$ have only one maximum at $E_{\text {c.m. }} \approx$ $V_{b}^{\text {orient }}$ (sphere-sphere $)=V_{b}$ as in the experiment [50]. With increasing increment the barrier distribution is shifted to 

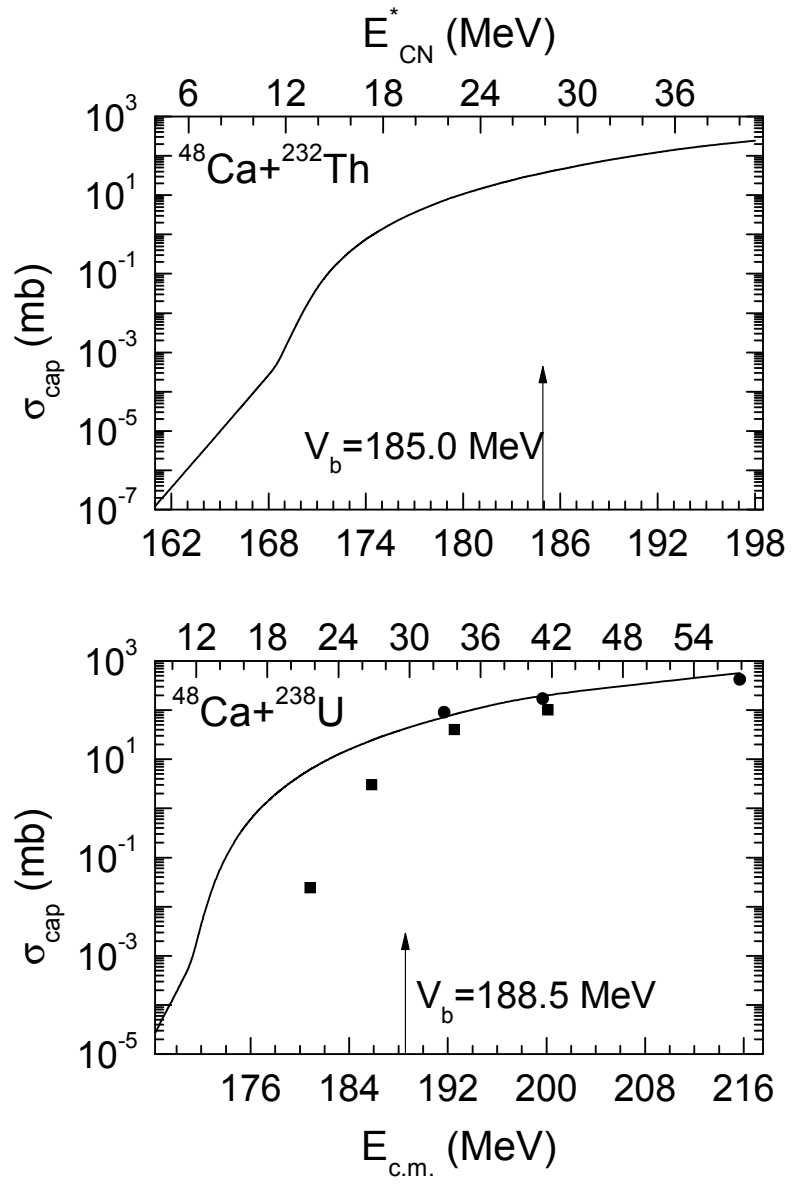

FIG. 13: The same as in Fig. 4, but for the ${ }^{48} \mathrm{Ca}+{ }^{232} \mathrm{Th},{ }^{238} \mathrm{U}$ reactions. The excitation energies $E_{C N}^{*}$ of the corresponding nuclei are indicated. The experimental data are taken from Refs. 48] (marked by squares) and 47] (marked by circles). The static quadrupole deformation parameters are: $\beta_{2}\left({ }^{238} \mathrm{U}\right)=0.286, \beta_{2}\left({ }^{232} \mathrm{Th}\right)=0.261$ and $\beta_{1}\left({ }^{48} \mathrm{Ca}\right)=0$.

lower energies. Assuming a spherical target nucleus in the calculations, we obtain a more narrow barrier distribution (see Fig. 12).

\section{Capture cross sections in reactions with large fraction of quasifission}

In the case of large values of $Z_{1} \times Z_{2}$ the quasifission process competes with complete fusion at energies near barrier and can lead to a large hindrance for fusion, thus ruling the probability for producing superheavy elements in the actinidebased reactions [45, 46]. Since the sum of the fusion cross section $\sigma_{f u s}$, and the quasifission cross section $\sigma_{q f}$ gives the capture cross section,

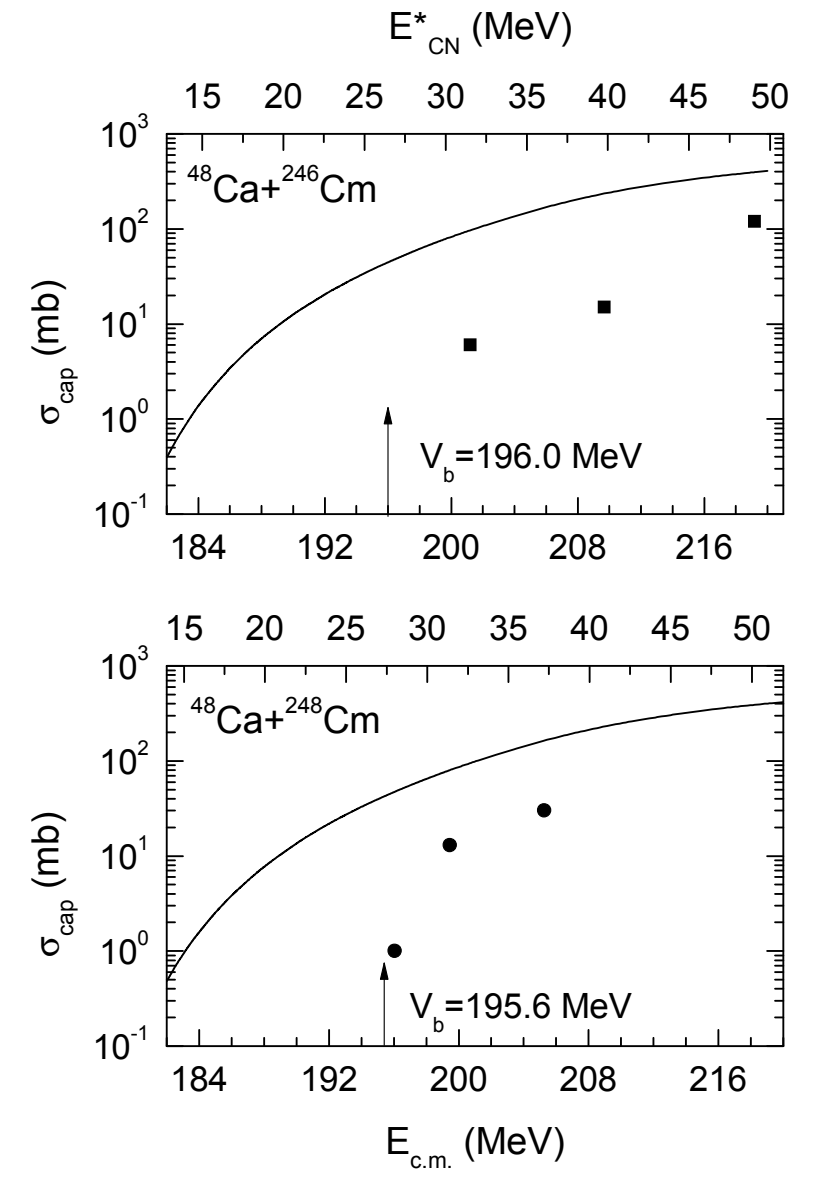

FIG. 14: The same as in Fig. 13, but for the reactions ${ }^{48} \mathrm{Ca}$ $+{ }^{246,248} \mathrm{Cm}$. The experimental data are from Refs. [48] (squares) and [49] (circles). The static quadrupole deformation parameters are: $\beta_{2}\left({ }^{246} \mathrm{Cm}\right)=0.298, \beta_{2}\left({ }^{248} \mathrm{Cm}\right)=0.297$ and $\beta_{1}\left({ }^{48} \mathrm{Ca}\right)=0$.

and $\sigma_{f u s} \ll \sigma_{q f}$ in the actinide-based reactions ${ }^{48} \mathrm{Ca}+$ ${ }^{232} \mathrm{Th},{ }^{238} \mathrm{U},{ }^{244} \mathrm{Pu},{ }^{246,248} \mathrm{Cm}$ and ${ }^{50} \mathrm{Ti}+{ }^{244} \mathrm{Pu}$ [46], we have

$$
\sigma_{c a p} \approx \sigma_{q f}
$$

In a wide mass-range near the entrance channel, the quasifission events overlap with the products of deep-inelastic collisions and can not be firmly distinguished. Because of this the mass region near the entrance channel is taken out in the experimental analyses of Refs. [48, 49]. Thus, by comparing the calculated and experimental capture cross sections one can study the importance of quasifission near the entrance channel for the actinide-based reactions leading to superheavy nuclei.

The capture cross sections for the quasifission reactions [4]49] are shown in Figs. 13-15. One can observe a large deviations of the experimental data of Refs. [48, 49] from the the calculated results. The possible reason is an underestimation of the quasifission yields measured in these reactions. Thus, the quasifission yields near the entrance channel are important. Note that there are the experimental uncertainties in

$$
\sigma_{c a p}=\sigma_{f u s}+\sigma_{q f}
$$




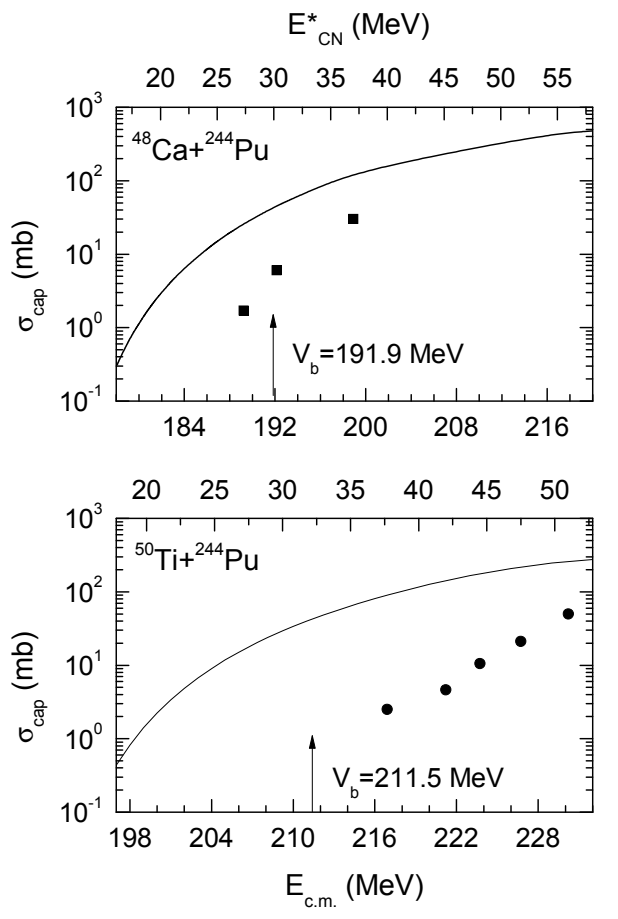

FIG. 15: The same as in Fig. 13, but for the indicated ${ }^{48} \mathrm{Ca},{ }^{50} \mathrm{Ti}+{ }^{244} \mathrm{Pu}$ reactions. The experimental data are from Refs. [49] (squares) and [48] (circles). The static quadrupole deformation parameters are: $\beta_{2}\left({ }^{244} \mathrm{Pu}\right)=0.293$, and $\beta_{1}\left({ }^{48} \mathrm{Ca}\right)=\beta_{1}\left({ }^{50} \mathrm{Ti}\right)=0$.

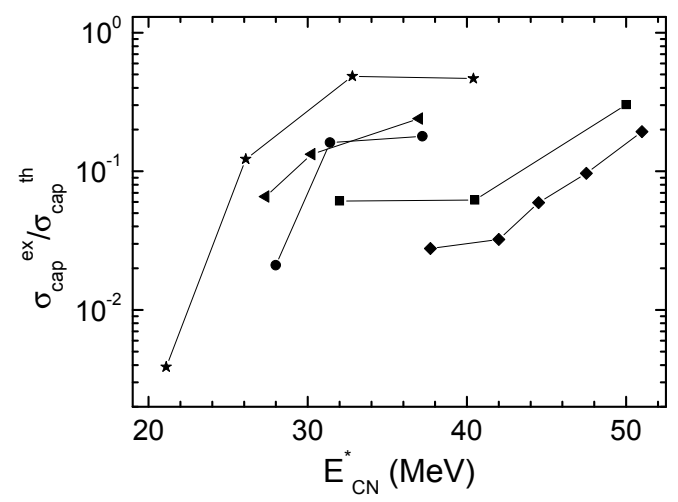

FIG. 16: The ratio of theoretical and experimental capture cross sections versus the excitation energy $E_{\text {c.m. }}$. of the compound nucleus for the reactions ${ }^{48} \mathrm{Ca}+{ }^{238} \mathrm{U}$ (closed stars), ${ }^{48} \mathrm{Ca}+{ }^{244} \mathrm{Pu}$ (closed triangles), ${ }^{48} \mathrm{Ca}+{ }^{246} \mathrm{Cm}$ (closed squares), ${ }^{48} \mathrm{Ca}+{ }^{248} \mathrm{Cm}$ (closed circles), and ${ }^{50} \mathrm{Ti}+{ }^{244} \mathrm{Pu}$ (closed rhombuses). the bombarding energies.

One can see in Fig. 16 that the experimental and the theoretical cross sections become closer with increasing bombarding energy. This means that with increasing bombarding energy the quasifission yields near the entrance channel massregion decrease with respect to the quasifission yields in other mass-regions. The quasifission yields near the entrance channel increase with $Z_{1} \times Z_{2}$.

\section{SUMMARY}

The quantum diffusion approach is applied to study the capture process in the reactions with deformed nuclei at subbarrier energies. The available experimental data at energies above and below the Coulomb barrier are well described, showing that the static quadrupole deformations of the interacting nuclei are the main reasons for the capture cross section enhancement at sub-barrier energies. Since the deformations of the interacting nuclei mainly influence the slope of curve at $E_{\text {c.m. }}<V_{b}$ and one can extract the ground state deformation of projectile or target from the experimental capture cross section data.

Due to a change of the regime of interaction (the turningoff of the nuclear forces and friction) at sub-barrier energies, the curve related to the capture cross section as a function of bombarding energy has smaller slope $E_{\text {c.m. }}-V_{b}<-5 \mathrm{MeV}$. This change is also reflected in the functions $\left\langle J^{2}\right\rangle, L\left(E_{\text {c.m. }}\right)$, and $S\left(E_{\text {c.m. }}\right)$. The mean-square angular momentum of captured system versus $E_{\text {c.m. }}$ has a minimum and then saturates at sub-barrier energies. This behavior of $\left\langle J^{2}\right\rangle$ would increase the expected anisotropy of the angular distribution of the products of fission and quasifission following capture. The astrophysical factor has a maximum and a minimum at energies below the barrier. The maximum of $L$-factor corresponds to the minimum of the $S$-factor. One can suggest the experiments to check these predictions.

The importance of quasifission near the entrance channel is shown for the actinide-based reactions leading to superheavy nuclei.

\section{ACKNOWLEDGEMENTS}

This work was supported by DFG, NSFC, and RFBR. The IN2P3-JINR, MTA-JINR and Polish-JINR Cooperation programs are gratefully acknowledged.
[1] H.Q. Zhang et al., Phys. Rev. C 42, 1086 (1990).

[2] H.Q. Zhang et al., Phys. Rev. C 49, 926 (1994).

[3] Yu.Ts. Oganessian et al., JINR Rapid Communications 75, 123 (1996).

[4] Z. Liu et al., Phys. Rev. C 54, 761 (1996).
[5] D.M. Nadkarni et al., Phys. Rev. C 59, R580 (1999).

[6] M. Trotta et al., Phys. Rev. Lett. 84, 2342 (2000).

[7] C.L. Jiang et al., Phys. Rev. Lett. 89, 052701 (2002).

[8] S.P. Tretyakova, A.A. Ogloblin, R.N. Sagaidak, S.V. Khlebnikov, and W. Trzaska, Nucl. Phys. 
A734, E33 (2004); S.P. Tretyakova, A.A. Ogloblin, R.N. Sagaidak, W. Trzaska, S.V. Khlebnikov, R. Julin, and J. Petrowski, Nucl. Phys. A738, 487 (2004).

[9] K. Nishio et al., Phys. Rev. Lett. 93, 162701 (2004).

[10] C.L. Jiang et al., Phys. Rev. C 71, 044613 (2005).

[11] K. Nishio et al., Eur. Phys. J. A 29 (2006).

[12] W. Loveland et al., Phys. Rev. C 74 (2006) 044604; A.M. Vinodkumar et al., Phys. Rev. C 74 (2006) 064612; A.M. Vinodkumar et al., Phys. Rev. C 78 (2008) 054608.

[13] M. Dasgupta et al., Phys. Rev. Lett. 99, 192701 (2007).

[14] K. Nishio et al., Phys. Rev. C. 77, 064607 (2008).

[15] D.J. Hinde et al., Phys. Rev. Lett. 101, 092701 (2008).

[16] M.G. Itkis et al., Nucl. Phys. A 834, 374c (2010).

[17] H.Q. Zhang et al., Phys. Rev. C. 81, 034611 (2010).

[18] K. Langanke and C.A. Barnes, Adv. Nucl. Phys. 22, 173 (1996).

[19] A. Aprahamian, K. Langanke, and M. Wiescher, Prog. Part. Nucl. Phys. 54, 535 (2005).

[20] L.E. Canto, P.R.S. Gomes, R. Donangelo, and M.S. Hussein, Phys. Rep. 424, 1 (2006).

[21] H. Esbensen and C.L. Jiang, Phys. Rev. C 79, 064619 (2009); S. Misicu and H. Esbensen, Phys. Rev. C 75, 034606 (2007); H. Esbensen and S. Misicu, Phys. Rev. C 76, 054609 (2007).

[22] K. Hagino and N. Rowley, AIP Conf. Proc. 1098, 18 (2009).

[23] V.V.Sargsyan, G.G. Adamian, N.V. Antonenko, and W. Scheid, Eur. Phys. J. A 45, 125 (2010).

[24] G.G. Adamian et al., Int. J. Mod. Phys. E 5, 191 (1996).

[25] S. Raman, C.W. Nestor, Jr, and P. Tikkanen, At. Data Nucl. Data Tables 78, 1 (2001).

[26] V.V. Dodonov and V.I. Man'ko, Trudy Fiz. Inst. AN 167, 7 (1986).

[27] H. Hofmann, Phys. Rep. 284, 137 (1997); C. Rummel and H. Hofmann, Nucl. Phys. A 727, 24 (2003).

[28] G.G. Adamian, N.V. Antonenko, Z. Kanokov, and V.V. Sargsyan, Teor. Mat. Fiz. 145, 87 (2005) [Theor. Math. Phys. 145, 1443 (2006)]; Z. Kanokov, Yu.V. Palchikov, G.G. Adamian, N.V. Antonenko, and W. Scheid, Phys. Rev. E 71, 016121 (2005);
Yu.V. Palchikov, Z. Kanokov, G.G. Adamian, N.V. Antonenko, and W. Scheid, Phys. Rev. E 71, 016122 (2005).

[29] N. Takigawa, S. Ayik, K. Washiyama, and S. Kimura, Phys. Rev. C 69, 054605 (2004).

[30] G. Hupin and D. Lacroix, Phys. Rev. C 81, 014609 (2010).

[31] V.V. Sargsyan, Z. Kanokov, G.G. Adamian, N.V. Antonenko, and W. Scheid, Phys. Rev. C 80, 034606 (2009); Phys. Rev. C 80, 047603 (2009).

[32] G.G. Adamian, A.K. Nasirov, N.V. Antonenko, and R.V. Jolos, Phys. Part. Nucl. 25, 583 (1994).

[33] K. Washiyama, D. Lacroix, and S. Ayik, Phys. Rev. C 79, 024609 (2009); S. Ayik, K. Washiyama, and D. Lacroix, Phys. Rev. C 79, 054606 (2009).

[34] K. Langanke and S.E. Koonin, Nucl. Phys. A410, 334 (1983); A. Redder, H.W. Becker, C. Rolfs, H.P. Trautvetter, T.R. Donoghue, T.C. Rinckel, J.W. Hammer, and K. Langanke, Nucl. Phys. A462, 385 (1987).

[35] J. Toke et al., Nucl. Phys. A 440, 327 (1985).

[36] V.E. Viola, Jr., and T. Sikkeland et al., Phys. Rev. 128, 767 (1962).

[37] B.B. Back et al., Phys. Rev. C. 32, 195 (1985).

[38] T. Murakami et al., Phys. Rev. C 34, 1353 (1986).

[39] S. Kailas et al., Phys. Rev. C 59, 2580 (1999).

[40] K. Nishio et al., Phys. Rev. C 82, 044604 (2010).

[41] P. Möller et al., At. Data Nucl. Data Tables 59, 185 (1995).

[42] R. Vandenbosch, Annu. Rev. Nucl. Part. Sci. 42, 447 (1992).

[43] A.B. Balantekin, J.R. Bennett, and S. Kuyucak, Phys. Lett. B 335, 295 (1994).

[44] C.Y. Wong, Phys. Rev. Lett. 31, 766 (1973).

[45] M. Trotta et al., Eur. Phys. J. A 25, 615 (2005).

[46] G.G. Adamian, N.V. Antonenko, and W. Scheid, Phys. Rev. C 68, 034601 (2003).

[47] W.Q. Shen et al., Phys. Rev. C 36, 115 (1987).

[48] M.G. Itkis et al., Nucl. Phys. A 787, 150c (2007).

[49] M.G. Itkis et al., Nucl. Phys. A 734, 136c (2004).

[50] D.J. Hinde et al., Phys. Rev. Lett. 74, 1295 (1995). 